00

\title{
Генерация третьей гармоники терагерцовых волн нелинейной графеновой многослойной метаповерхностью
}

\author{
(C) А.М. Лерер ${ }^{1}$, Г.С. Макеева ${ }^{2}$, В.В. Черепанов ${ }^{1}$ \\ ${ }^{1}$ Южный федеральный университет, физический фракультет, \\ 344090 Ростов-на-Дону, Россия \\ ${ }^{2}$ Пензенский государственный университет, \\ 440026 Пенза, Россия \\ e-mail: lerer@sfedu.ru; radiotech@pnzgu.ru
}

Поступила в редакцию 07.06.2020 г.

В окончательной редакции 07.06.2020 г.

Принята к публикации 08.09.2020 г.

В терагерцовом $(\mathrm{THz})$ диапазоне исследована нелинейная дифракция плоской электромагнитной волны на многослойных нелинейных метаповерхностях, состоящих из двумерно-периодических решеток прямоугольных графеновых лент конечной длины на разделительных диэлектрических слоях. Показано увеличение на несколько порядков эффективности генерации третьей гармоники на частотах резонанса поверхностного плазмон-поляритона. Показано, что частоту генерации третьей гармоники можно регулировать путем динамической перестройки химического потенциала без изменения геометрии и размеров нелинейной графеновой метаповерхности.

Ключевые слова: нелинейная дифракция, многослойные метаповерхности, графеновые ленты, генерация третьей гармоники, поверхностный плазмон-поляритон.

DOI: $10.21883 /$ OS.2021.01.50444.173-20

Нелинейные свойства графена делают его привлекательным материалом для нелинейных оптоэлектронных приложений на ТГц частотах [1,2]. Экспериментальные результаты иллюстрируют отчетливые нелинейные свойства графена [2], которые могут стать основой для усовершенствования нелинейных и сверхбыстродействующих нанофотонных устройств.

В работах [1,3-5] теоретически исследуется возможность генерации третьей гармоники на однослойных метаповерхностях, состоящих из решеток графеновых нанолент. При этом показано, что нелинейный отклик метаповерхности будет доминировать только от наиболее сильной керровской нелинейной восприимчивости графена, и поля в основном локализованы вдоль поверхности графеновых нанолент и значительно в меньшей степени внутри диэлектрического слоя [1].

Однако нелинейные процессы обычно имеют чрезвычайно слабую природу и требуют высокой входной мощности для возбуждения. Чтобы преодолеть это серьезное ограничение и значительно увеличить эффективность нелинейных взаимодействий в ТГц диапазоне частот, в настоящей работе предлагаются новые конструкции ультратонких многослойных нелинейных метаповерхностей, состоящих из двух-периодических решеток прямоугольных графеновых лент конечной длины на диэлектрических разделительных слоях.

В работе рассматривается только генерация третьей гармоники, а все другие нелинейные процессы, например, генерация высших гармоник, не учитываются, поскольку их генерируемые частоты отделены от частоты третьей гармоники.
Исследуемые метаповерхности - двумернопериодические решетки, образованные прямоугольными графеновыми, металлическими и диэлектрическими полосками (лентами конечной длины). Число полосок в трехмерной элементарной ячейке $M$, их размещение произвольное, т.е. они могут лежать как на поверхности верхнего слоя, так и в глубине. Число слоев диэлектрика $N_{l}$; слои могут быть с потерями, в том числе из плазмонных материалов. Числа $N_{l}, M-$ произвольные, практически они ограничены только параметрами компьютера.

Полагаем, что полоски бесконечно тонкие, и на них выполняются граничные условия - непрерывность тангенциальных компонент вектора напряженности электрического поля $\mathbf{E}_{\mathrm{tg}}$ и $\mathbf{j}=\sigma \mathbf{E}_{\mathrm{tg}}$, где $\mathbf{j}-$ поверхностная плотность тока, $\sigma$ - поверхностная проводимость. Модель поверхностной проводимости $\sigma$ графена как нелинейная функция напряженности электрического поля $\mathbf{E}$

$$
\sigma=\sigma_{l}+\chi|\mathbf{E}(x, y, t)|^{2},
$$

где $\sigma_{l}$ - линейная часть проводимости, определяется формулой Кубо [6].

Параметр $\chi$ определяется несколькими факторами. При комнатной температуре и условии бесстолкновительного приближения для носителей заряда параметр [7]:

$$
\chi=-i \frac{3 e^{4} v_{\mathrm{F}}}{32 \omega^{3} \hbar^{2} \mu_{c}},
$$

где $e-$ заряд электрона, $\mu_{c}-$ химический потенциал (уровень Ферми), $v_{\mathrm{F}}=10^{6} \mathrm{~m} / \mathrm{s}-$ скорость Ферми, $\omega-$ 
частота падающей волны, $\hbar-$ приведенная постоянная Планка. Из (2) видно, что изменением химического потенциала $\mu$ можно увеличить параметр $\chi$ и получить максимальный нелинейный отклик графена (1) и, как следствие, добиться эффективности нелинейного взаимодействия, в том числе максимального уровня генерации третьей гармоники.

Однако в низкочастотной части ТГц диапазона малая энергия фотонов и, следовательно, невысокая плотность потока мощности падающей волны накладывают некоторые ограничения на значения химического потенциала [8]. Химический потенциал следует выбирать из условия $\mu \gg k_{B} T$. При комнатной температуре и частотах порядка $1 \mathrm{THz} \mu_{c} \gg 0.26 \mathrm{eV}$.

Математическая модель

- Определяются поля в многослойном диэлектрике при произвольном расположении источников планарных токов по методу, описанному в [9];

- выполняются граничные условия на полосках при $\chi=0$ в (1);

- результат этих этапов - система парных интегральных уравнений (ПИУ) относительно плотностей токов на полосках $\mathbf{j}$; эти ПИУ близки к ПИУ [10], но в [10] ПИУ только для одной полоски;

- система ПИУ решается методом Галеркина как в ПИУ [10] для одной полоски;

- рассчитываются поля как в дальней зоне, так и на полосках;

- рассчитываются нелинейные токи на полосках и по формулам, полученным на первом этапе, находим амплитуды прошедшей и отраженной от метаповерхности волн с утроенной частотой.

Рассмотрим результаты исследования нелинейной дифракции нормально падающей плоской однородной электромагнитной волны (ТЕМ-волна) на многослойной метаповерхности, состоящей из трех двух-периодических решеток (периодичность $d_{x}=d_{y}=35 \mu \mathrm{m}$ ) прямоугольных лент графена конечной длины, расположенных на диэлектрических слоях $n=1.45\left(\mathrm{SiO}_{2}\right)$, толщиной $d_{1}=d_{2}=30 \mu \mathrm{m}$ и $d_{3}=15 \mu \mathrm{m}$ на полубесконечной подложке $n=1.77\left(\mathrm{Al}_{2} \mathrm{O}_{3}\right)$. Нумерация слоев сверху вниз. Графеновые ленты на поверхности слоев расположены друг под другом.

Обозначения поляризации падающей волны как в оптике: $s(p)$-поляризация. При $s$-поляризации вектор напряженности электрического поля ориентирован вдоль широкой стороны полосок. При $p$-поляризации - вдоль узкой стороны полосок.

На рис. 1 приведены результаты моделирования частотных зависимостей коэффициентов отражения $R$, прохождения $T$ (по мощности) и коэффициента поглощения $P$ при дифракции ТЕМ-волны (линейный режим).

Поля падающей волны локально ограничены по поверхности графена (в большей степени, чем внутри диэлектрических разделительных слоев) и резко возрастают при резонансе за счет возбуждения стоячих волн поверхностных плазмон-поляритонов вдоль графеновых

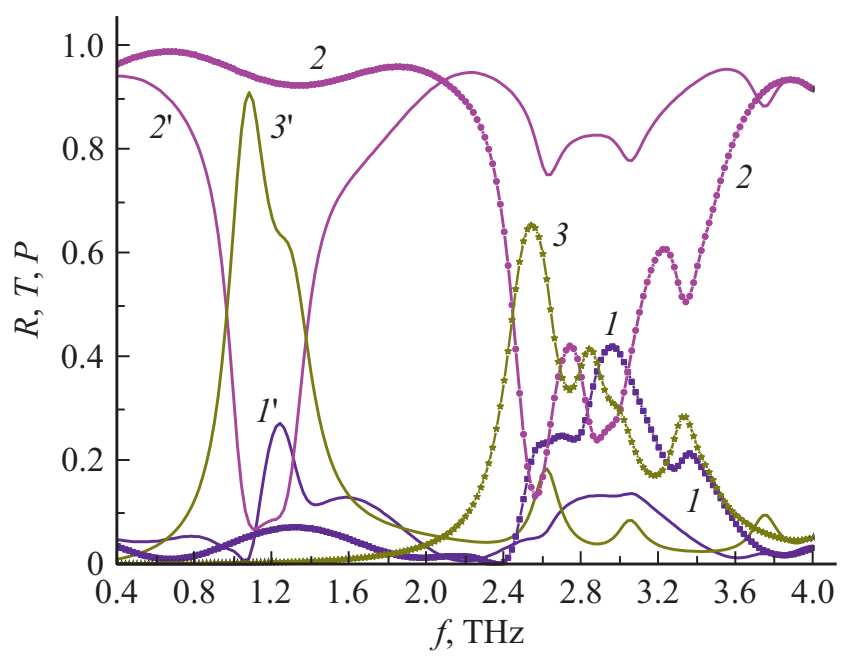

Рис. 1. Частотные зависимости коэффициентов отражения $R$ (кривые $\left.1,1^{\prime}\right)$, прохождения $T\left(2,2^{\prime}\right)$ и поглощения $P\left(3,3^{\prime}\right)$ падающей волны от многослойной графеновой метаповерхности. Линии с символами $(1,2,3)-p$-поляризация, без символов $\left(1^{\prime}, 2^{\prime}, 3^{\prime}\right)-s$-поляризация. Размеры лент $10 \times 25 \mu \mathrm{m}^{2}$. Параметры графена $\mu_{c}=0.35 \mathrm{eV}, \tau=1 \mathrm{ps}, T=300 \mathrm{~K}$.

нанолент. Кроме того, эффекты стоячей волны, основанные на резонансах Фабри-Перо (благодаря отражению от диэлектрических разделительных слоев, подложки и графеновых решеток), вызывают дальнейшее увеличение электрического поля и более острый резонансный отклик на моде входной основной частоты. Для усиления этого эффекта в работе проведена оптимизация толщин диэлектрика $d_{1}, d_{2}, d_{3}$.

Резонанс зависит от поляризации падающей волны. Для $s$-поляризации резонансная частота определяется основной модой поверхностных плазмон-поляритонов (резонансом тока вдоль широкой стороны полосок). Для $p$-поляризации - резонанс тока вдоль узкой стороны полосок, и резонансная частота больше, чем при $s$ поляризации (рис. 1). Наблюдается несколько резонансных частот (три) ближайших высших мод поверхностных плазмон-поляритонов (рис. 1).

На рис. 2 приведены результаты моделирования частотных зависимостей нормированной мощности третьей гармоники, излучаемой в прямом направлении $T_{3}$ (прохождение) и обратном направлении $R_{3}$ (отражение), при нелинейной дифракции на многослойной графеновой метаповерхности как функции частоты падающей волны для волн $s$-, $p$-поляризации. Значения $T_{3}, R_{3}$ нормированы на плотность мощности падающей волны $10 \mathrm{~mW} / \mathrm{mm}^{2}$. На оси абсцисс $f$ - частота падающей волны. Видно, что эффективность генерации третьей гармоники увеличивается на несколько порядков по амплитуде, когда частота падающей волны близка к резонансу графеновой метаповерхности (рис. 2), так как поля на резонансной частоте сильно ограничены и значительно увеличены вдоль поверхности лент графена за 


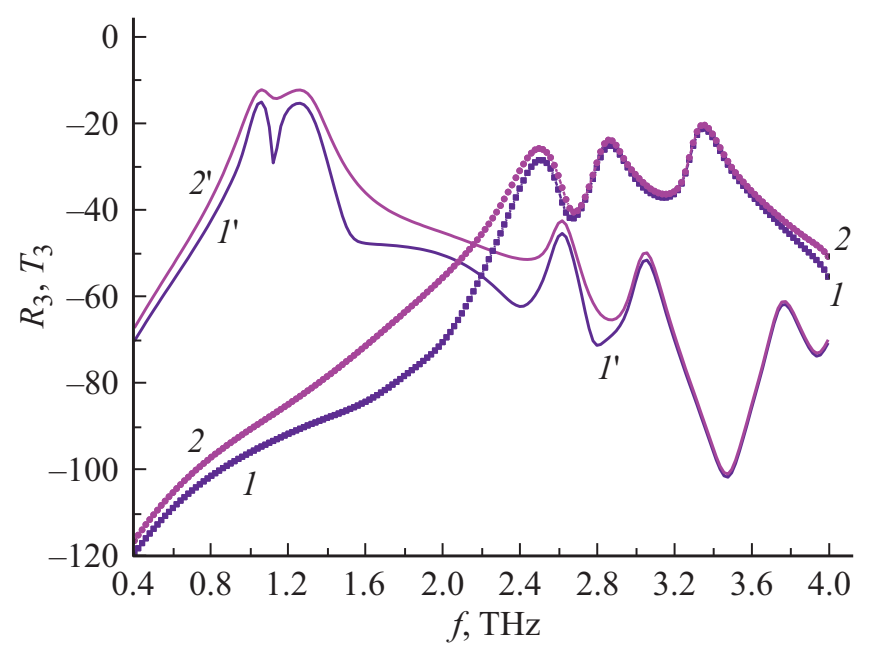

Рис. 2. Нормированная мощность 3-й гармоники, излучаемой в обратном направлении $R_{3}$ (отражение) и в прямом направлении $T_{3}$ (прохождение) при нелинейной дифракции на многослойной графеновой метаповерхности как функция частоты падающей волны. Кривые $1,1^{\prime}-R_{3}, 2,2^{\prime}-T_{3}$. Размеры лент и параметры графена указаны на подписи к рис. 1 .

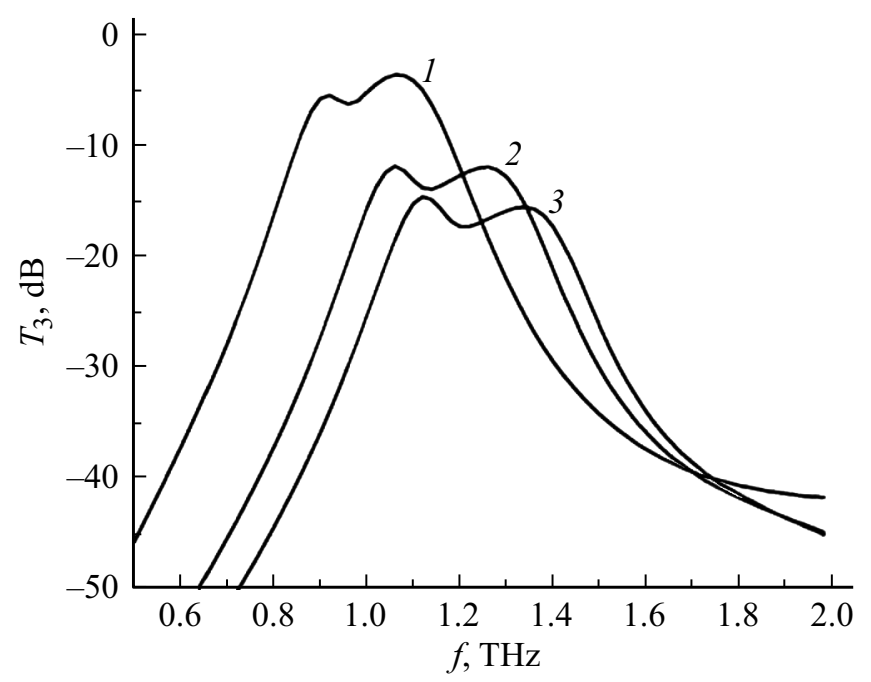

Рис. 3. Нормированная мощность 3-й гармоники, излучаемой в прямом направлении $T_{3}$ (прохождение) при нелинейной дифракции как функция частоты падающей $s$ поляризованной волны при разных значениях химического потенциала. $\mu_{c}=0.25 \mathrm{eV}-(1), 0.35$ (2), $0.4 \mathrm{~V}$ (3). Размеры графеновых лент $8 \times 25 \mu \mathrm{m}^{2}, \tau=1 \mathrm{ps}, T=300 \mathrm{~K}$.

счет возбуждения локализованных ТГц поверхностных плазмон-поляритонов.

Использование многослойных подложек (диэлектрического зеркала) позволяет управлять характеристиками метаповерхностей. Например, квазипериодическая подложка позволяет подавить прохождение волны первой гармоники, не уменьшая при этом излучаемой мощности третьей гармоники в прямом направлении $T_{3}$.
Резонансная частота графеновой метаповерхности может регулироваться путем динамической перестройки химического потенциала (энергии Ферми) графена с помощью электрического поля или химического легирования.

На рис. 3 приведены частотные зависимости $T_{3}, R_{3}$ (нелинейный режим) при разных значениях химического потенциала. Уменьшение приводит к уменьшению резонансной частоты и значительному увеличению эффективности генерации третьей гармоники. Однако дальнейшее уменышение может привести к некорректности использования (2).

Таким образом, с помощью разработанной математической модели нелинейного взаимодействия электромагнитных волн с многослойными графеновыми метаповерхностями показана возможность повысить эффективность генерации третьей гармоники на резонансных частотах поверхностного плазмон-поляритона в нижней части ТГц диапазона. Показано, что динамическая перестройка химического потенциала графена позволяет управлять резонансной частотой и уровнем генерации третьей гармоники в сверхширокой полосе частот. Предложенные многослойные нелинейные графеновые метаповерхности обеспечивают новый способ реализации компактных и эффективных электрически управляемых генераторов и широкополосных преобразователей частоты для применений в оптической связи и обработке ТГц сигналов, нелинейной ТГц спектроскопии и неинвазивных ТГц процессорах.

\section{Конфликт интересов}

Авторы заявляют, что у них нет конфликта интересов.

\section{Список литературы}

[1] Jin B., Guo T., Argyropoulos C. // J. Optics. 2017. V. 19. P. 094005.

[2] Jiang T., Kravtsov V., Tokman M., Belyanin A., Raschke M.B. // Nature Nanotechnology. 2019. V. 14. P. 838.

[3] Nasari H., Abrishamian M.S. // Nano Lett. 2016. V. 16. P. 2734.

[4] Savostianova N.A., Mikhailov S.A. // Appl. Phys. Lett. 2015. V. 107. P. 181104.

[5] Jadidi M.M., Kónig-Otto J.C., Winner S., Sushkov A.B., Drew H.D., Murphy T.E., Mittendorff M. // Nano Lett. 2016. V. 16. P. 2734. doi 10.1021/acs.nanolett.6b00405

[6] Hanson G.W. // J. Appl. Phys. 2008. V. 103. P. 064302.

[7] Pitilakis A., Chatzidimitriou D., Kriezis E.E. // Opt. Quant. Electron. 2016. V. 48. P. 243.

[8] Mikhailov S.A. // Europhysics Lett. 2007. V. 79. P. 27002.

[9] Лерер A.M. // РЭ. 2012. Т. 57. № 11. С. 1160. doi 10.1134/S106422691210004X

[10] Лерер А.М., Иванова И.Н. // РЭ. 2016. Т. 61. № 5. С. 435. doi $10.1134 / \mathrm{S} 1064226916050089$ 